\title{
Comparative analysis of phenotypic traits in two common genetic variants of limb-girdle muscular dystrophy
}

\author{
I.V. Sharkova ${ }^{1}$, E. L. Dadali ${ }^{1}$ I.V. Ugarov ${ }^{2}$, O.P. Ryzhkova ${ }^{1}$, A.V. Polyakov ${ }^{1}$ \\ ${ }^{1}$ Federal State budgetary institution «Research centre for medical genetics»; 1 Moskvorechye St., Moscow, 115478, Russia; \\ ${ }^{2}$ Department of Urology, A.I. Evdokimov Moscow State University of Medicine and Dentistry; \\ 1 Bldg, 20 Delegatskaya St., Moscow, 127473, Russia
}

Контакты: Инна Валентиновна Шаркова sharkova-inna@rambler.ru

The algorithm of differential diagnosis of two most common genetic limb-girdle muscular dystrophy variants (LGMD2A and DBPMD), developed on the basis of a comprehensive survey of 85 patients with specification of diagnosis by using techniques of DNA analysis. It is shown that the accurate diagnosis of LGMD genetic types should be based on the results of the clinical and genealogical, biochemical and molecular genetic analysis. The proposed algorithm significantly reduces the economic and time costs with expensive DNA testing.

Key words: limb-girdle muscular dystrophy, Duchenne/Becker progressive muscular dystrophy, calpainopathy, dystrophinopathies, CAPN3 gene, DMD gene, large joints contractures, calf muscles pseudohypertrophy, medical genetic counseling, algorithms of the diagnosis

DOI: $10.17650 / 2222-8721-2015-5-3-42-48$

\section{Introduction}

limb-girdle muscular dystrophy (LGMD) is a group of clinically and genetically heterogeneous polymorphic diseases characterized by progressive muscle weakness, atrophy of skeletal muscles with the primary lesion of the shoulder and pelvic girdle, decreased tendon reflexes, increased blood levels of creatine phosphokinase (CK) [1-5].

To the current date there are 31 genetic variants of LGMD that occur after normal motor development period caused by mutations in the genes localized in autosomes [6]. There are 3 genetic variants with $\mathrm{X}$-linked recessive mode of inheritance - Duchenne/Becker progressive muscular dystrophy (DBPMD) and Emery-Dreifuss muscular dystrophy (EDMD) type 1 and 6 [7].

The incidence of all LGMD variants vary in different populations from 5 to 70 patients per 1 million populations [8].

DBPMD is the most common in this group of diseases. Its prevalence is $1: 3500$ of live male births $[9,10]$, while LGMD type 2A (LGMD2A) accounts for 30 to $40 \%$ of all LGMD cases with autosomal-recessive inheritance pattern $[8,11,12]$.

LGMD2A (OMIM: 253600) is caused by mutations in calpain 3 (CAPN3) gene located in 15q15.1-q21.1 [13]. The product of its expression is an enzyme from the family of calcium-dependent proteases. The latter is involved in synchronization of muscle contraction, in the process of miofibrillogenesis as well as sarcomeric remodeling $[14,15]$. Being two allelic variants Duchenne LGMD (OMIM: 310200 ) and Becker LGMD (OMIM: 300376) have specific clinical pictures due to different mutations in dystrophin gene (DMD), located on the short arm of X chromosome in r21.2-21.3 [16-19]. The product of DMD gene is a structural protein which is a part of dystrophin-glycoprotein complex. The latter helps to connect cytoskeleton of myofibers to the extracellular matrix [20]. Therefore, both expression products of these genes are involved in providing synchronism of the complex process of muscle contraction. Therefore, the similarity of clinical manifestations of these genetic LGMD variants is caused by participation of gene expression products in a single pathogenic mechanism [21, $22]$. This fact greatly complicates their differential diagnosis in the clinical stage of the survey and makes it impossible to prevent the emergence of repeated cases in families with burdened anamnesis.

The purpose of the work was to identify particular phenotypic manifestations that allow their differential diagnosis in the clinical stage of the survey based on the analysis of the frequency of 33 clinical signs in samples of patients with DBPMD and LGMD2A.

\section{Materials and methods}

Analysis of phenotypic traits in 85 patients (64 men and 21 women) from 82 families aged from 3 to 58 years with clinical manifestations of LGMD that develops after the period of normal motor development.

Based on the results of DNA analysis we formed two groups of LGMD patients depending on its etiology. The first group consisted of 45 male DBPMD patients, while group 2 consisted of 40 LGMD2A patients (19 men and 21 women).

Molecular genetic analysis of the DNA samples was carried out in DNA diagnostics lab of Medical Genetic Science Center. Isolation of genomic DNA from peripheral blood leukocytes in patients was carried out by using a set of reagents for DNA isolation called Prep100 (DIA- 
tom $^{\mathrm{TM}}$ ) according to the manufacturer»s protocol. CAPN3 gene was studied by direct automated sequencing method of coding regions, including exon-intron junctions, DMD gene by the method of multiplex amplification with analysis of 20 exons and promoter region.

Phenotype map was prepared for each patient. It included 2 quantitative signs (age of onset of the disease and the level of CK activity in the blood plasma) and 31 qualitative signs.

Here is a list of clinical symptoms and signs, used to describe the phenotype of LGMD2A and DBPMD patients.

1. Age of onset below 5 years.

2. Age of onset 6 to 10 years.

3. Age of onset 11-20 years.

4. Age of onset over 20 years.

5. Pterygoid blades.

6. Flabby shoulder girdle symptom.

7. Wasp waist.

8. Waddling gait.

9. Steppage gait.

10. Difficulty in climbing stairs.

11. Gower»s maneuver.

12. Violation of walking on heels.

13. Violation of walking on toes.

14. Hypotrophy of shin muscles.

15. Hypotrophy of forearm muscles.

16. Hypotrophy of rotator girdle muscles.

17. Hypotrophy of pelvic girdle muscles.

18. Lack/reduced Achilles reflex.

19. Lack/reduced knee reflex.

20. Lack/reduced biceps reflex.

21. Lack/reduced carporadial reflex.

22. Reduced forces in the distal portions of the lower extremities.

23. Reduced forces in the distal portions of the upper extremities.

24. Reduced forces in the proximal portions of the lower extremities.

25. Reduced forces in the proximal portions of the upper extremities.

26. Feet deformations.

27. Hand deformations.

28. Contractures of major joints.

29. Contracture of minor joints.

30. Lumbar hyperlordosis.

31. Scoliosis.

32. Cardiomyopathy.

33. CK level.

34. Diffuse muscular hypotonia.

35. Pseudohypertrophy.

36. Asymmetry of the lesion.

Statistical analysis of the results was aimed at identifying the core of clinical features, the incidence of which has statistically significant differences in 2 groups of LGMD patients. Results of the clinical studies were numerically expressed in a binary rating scale, i. e. 1 of 2 possible values were assigned to each clinical sign: $\mathrm{Xi}=1$ if the corresponding sign was observed in patient, and $\mathrm{Xi}=0$ in the contrary case. Thus, phenotype of each of the patients in the study groups was expressed as a point in the multidimensional space of clinical signs. Coordinates of the point were equal to specific characteristic values. Accordingly, the description of each of the clinical phenotype of test groups is the set of points, each of which expresses a clinical phenotype of the individual patient. Clinical phenotype of the study groups is presented in the form of the confidence sphere of a certain radius. Centre of the sphere is a point with coordinates determined as arithmetic mean values of each of the sign, calculated for all patient in the group. Radius of the sphere is that one in which confidence sphere «absorbs» $95 \%$ of the points belonging to a particular group.

Significance level of 0.05 was used when comparing an incidence of clinical signs, the differences were considered significant at $p<0.05$. Comparison of frequencies was performed by the standard method of comparison of sample fractions. Statistical significance of differences in the prevalence of clinical signs in certain groups of patients was based on Student»s t-test for sampled frequencies of signs, taking into account Yates» correction for continuity. Nonparametric Mann-Whitney criterion was used to analyze differences between the groups in the age of onset as the nature of distribution of the values differs from normal one in the studied groups. Comparison of treatment groups by $\mathrm{CK}$ values (measured in a quantitative scale) was performed by using nonparametric Kruskal-Wallis test for the analysis of multiple differences. The necessity to use non-parametric test is caused by the fact that the distribution of CK values in two compared groups differs from the normal ones, which makes it impossible to compare the study groups by CK values based on the analysis of variance.

\section{Results and discussion}

Frequency analysis of clinical signs revealed high degree of similarity between the phenotypes of LGMD patients in both study groups - DBPMD and LGMD2A. As was mentioned above, this may be caused by the similarity of pathogenetic mechanisms of the discussed diseases. Considerable overlapping of regions characterizes the variability of clinical phenotype in LGMD patients of the analyzed groups (Fig. 1). This indicates the proximity of age of onset of the studied LGMD types. At the same time in each of the analyzed group there are fragments of spheres that do not have overlapping region corresponding to different LGMD type. This indicates the presence of certain symptoms that can be used for differential diagnosis of DBPMD and LGMD2A at the clinical level. Frequency analysis of different symptoms in the studied groups of DBPMD and LGMD2A patients revealed significant differences of the following signs: pterygoid blades, flabby shoulder girdle symptom, wasp waist, waddling gait, difficulty in climbing stairs, lumbar hyperlordosis, diffuse muscular hypotonia, 


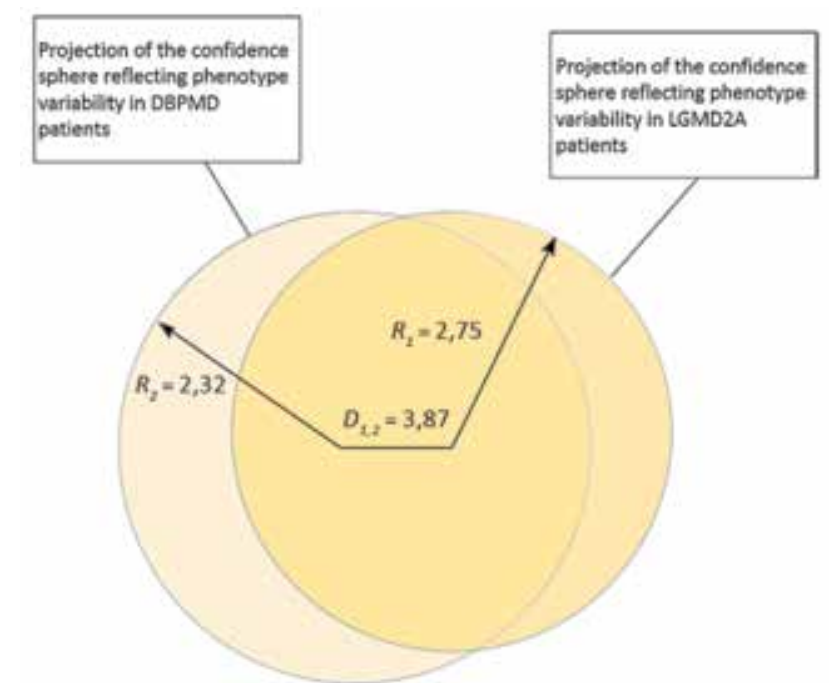

Fig. 1. Projections of the confidence spheres reflecting phenotype variability in DBPMD and LGMD2A patients

pseudohypertrophy of the calf muscles (see the table). All of these signs were found significantly more common in DBPMD patients.

Violation of walking on heels is significantly more common in LGMD2A patients, as well as hypertrophy of lower leg muscles, muscles of the shoulder and pelvic girdle, reduction of force in the proximal portion of upper limbs, contractures of large joints, scoliosis.

Although above-listed clinical signs have significant differences in the frequency of occurrence in the studied LGMD forms, their presence does not necessarily reflects nosological specificity. For example, pseudohypertrophy of the calf muscles was found in $93.3 \%$ of DBPMD patients, but in $6.7 \%(n=3)$ of patients in the same group we revealed hypotrophy of lower leg muscles. The presence of hypo-, and atrophic changes of the lower leg muscles in DBPMD patients depends on the moment of examination of the patient with respect to the age of onset of the disease and is caused by the development of atrophic processes in the later stages of the disease in all muscle groups including calves.

At the same time, we revealed pseudohypertrophy of the calf muscles in $25 \%$ of LGMD2A patients, which generally coincides with the literature data confirming the presence of increased volume of calf muscles in $\geq 30 \%$ of patients with this genetic variant [23, 24]. In all cases of calf muscles pseudohypertrophy in patients with negative DNA test for DBPMD one should be alert to the possible genetic defect of calpain.

Contractures of major joints were detected in $62 \%$ of LGMD2A patients. The ankle joints were primarily involved in the pathological process at the early stages of the disease, and the typical walking on toes developed. In the group of DBPMD patients contractures of major joints were 6 -fold less likely, but still occurred in $11 \%$ of patients, and in all cases were detected in the later stages of the disease.

Contracture of minor joints, asymmetry of the lesion, deformation of hands and feet in LGMD2A patients are rare conditions $(12.5 ; 5.0 ; 2.5$ and $2.5 \%$, respectively) and they did not occur in DBPMD group.

Thus, by comparing the frequency of clinical symptoms in DBPMD and LGMD2A patients one may conclude that there is a significant similarity of the clinical manifestations of these conditions. The observed clinical polymorphism in LGMD patients may be caused by a single pathogenic process, and a wide range of age of their manifestation, duration of the disease at time of examination of the patients of the surveyed samples.

Thus, in DBPMD group first signs of the disease occurred predominantly ( $91 \%$ ) below 5 years, while in the period from 6 to 10 years the first clinical manifestations were observed in $6.7 \%$ of the patients, and only in $2 \%$ of patients age of onset was in the period from 11 to 20 years. In LGMD2A group age of onset ranged more widely and occurred in all age ranges with a clear predominance in the period from 11 to 20 years $(57.5 \%)$ and from 6 to 10 years (27.5\%). Interestingly, in DBPMD patients there was not a single case of onset of the disease over 20 years, while in $10 \%$ of LGMD2A patients the disease manifested in this age period. The limited patient»s sample observed did not allow us to form a subgroup of a similar age of age of onset in the studied groups. The carried out comparison showed more significant results as the severity of the clinical manifestations of LGMD depend on the age of onset and duration of the course of the disease.

CK blood level can serve as an important criterion for the differential diagnosis of LGMD2A and DBPMD. When carrying out a comparative analysis of the average values of the level of CK activity in the blood serum in 2 LGMD groups, we found a statistically significant difference: LGMD2A - 3492.45 \pm 2828.01, DBPMD - 8687.95 \pm 6517.19 , Kruskal-Wallis criterion -7.48 at the significance level of $1.2676 \times 10^{-7}$ (Fig. 2).

It was shown that a typical feature of DBPMD is significant increase in CK level of at least 10-20 fold (often 50-fold, and in some cases up to 200-fold) of the upper limit of normal at the age below 5 years. High CK levels are observed in this group of patients at birth. In some studies, it is suggested that the increase in blood CK level less than 10-fold during the first 3 years of life in a child with suspected DBPMD should serve as an occasion for the diagnosis of other LGMD genetic variants [25]. According to the literature, peak value of this index is in the age period from 2 to 5.8 years. With an increasing age of the patient and progressive destruction of muscle fibers one can see a significant decrease in CK level, which does not allow to use this figure as an unambiguous diagnostic marker of particular LGMD genetic variant [26-28].

Thus, despite the identified significant differences in the incidence of individual signs and symptoms in DBPMD and LGMD2A patients, the analysis shows significant difficulties in diagnosis of these genetic variants on the clinical stage. However, we managed to identify a number of indica- 
Frequency of clinical signs among DBPMD and LGMD2A patients

\begin{tabular}{|c|c|c|c|c|}
\hline \multirow{2}{*}{ Sign } & \multicolumn{2}{|c|}{ Frequency, \% } & \multirow{2}{*}{$\mathbf{Q}$} & \multirow{2}{*}{$\mathbf{P}$} \\
\hline & LGMD2A & DBPMD & & \\
\hline Age of onset below 5 years & 91.1 & 2.5 & 35 & 0 \\
\hline Age of onset 6 to 10 years & 6.7 & 27.5 & 4.571429 & 0.03251 \\
\hline Age of onset $11-20$ years & 2.2 & 57.5 & 20.16667 & 0.000007 \\
\hline Age of onset over 20 years & 0 & 10 & 4 & 0.045501 \\
\hline Pterygoid blades & 97.8 & 55 & 17 & 0.000037 \\
\hline Flabby shoulder girdle symptom & 100 & 45 & 22 & 0.000003 \\
\hline Wasp waist & 75.6 & 5 & 27 & 0 \\
\hline Waddling gait & 100 & 55 & 18 & 0.000022 \\
\hline Steppage gait & 8.9 & 5 & 0.666667 & 0.414217 \\
\hline Difficulty in climbing stairs & 100 & 82.5 & 7 & 0.008151 \\
\hline Gower»s maneuver & 100 & 92.5 & 3 & 0.083265 \\
\hline Violation of walking on heels & 6.7 & 65 & 19.59259 & 0.00001 \\
\hline Violation of walking on toes & 2.2 & 5 & 0.333333 & 0.563703 \\
\hline Hypotrophy of shin muscles & 6.7 & 27.5 & 5.333333 & 0.020922 \\
\hline Hypotrophy of forearm muscles & 8.9 & 20 & 1.6 & 0.205904 \\
\hline Hypotrophy of rotator girdle muscles & 26.7 & 92.5 & 21.55172 & 0.000003 \\
\hline Hypotrophy of pelvic girdle muscles & 26.7 & 92.5 & 21.55172 & 0.000003 \\
\hline Lack/reduced Achilles reflex & 80 & 82.5 & 0.090909 & 0.763025 \\
\hline Lack/reduced knee reflex & 86.7 & 92.5 & 0.5 & 0.479501 \\
\hline Lack/reduced biceps reflex & 73.3 & 85 & 1.666667 & 0.196707 \\
\hline Lack/reduced carporadial reflex & 73.3 & 67.5 & 0.25 & 0.617075 \\
\hline Reduced forces in the distal portions of the lower extremities & 48.9 & 42.5 & 0.6 & 0.438579 \\
\hline Reduced forces in the distal portions of the upper extremities & 28.9 & 30 & 0.076923 & 0.781511 \\
\hline $\begin{array}{l}\text { Reduced forces in the proximal portions of the lower } \\
\text { extremities }\end{array}$ & 100 & 97.5 & 1 & 0.317311 \\
\hline $\begin{array}{l}\text { Reduced forces in the proximal portions of the upper } \\
\text { extremities }\end{array}$ & 71.1 & 97.5 & 7.363636 & 0.006656 \\
\hline Feet deformations & 0 & 12.5 & 5 & 0.025348 \\
\hline Hand deformations & 0 & 2.5 & 1 & 0.317311 \\
\hline Contractures of major joints & 11.1 & 62.5 & 18.18182 & 0.00002 \\
\hline Contracture of minor joints & 0 & 2.5 & 1 & 0.317311 \\
\hline Lumbar hyperlordosis & 100 & 42.5 & 23 & 0.000002 \\
\hline Scoliosis & 2.2 & 22.5 & 6.4 & 0.011413 \\
\hline Cardiomyopathy & 17.8 & 25 & 1 & 0.317311 \\
\hline Diffuse muscular hypotonia & 100 & 52.5 & 19 & 0.000013 \\
\hline Pseudohypertrophy of the calf muscles & 93.3 & 25 & 27 & 0 \\
\hline Asymmetry of the lesion & 0 & 5 & 2 & 0.1573 \\
\hline
\end{tabular}




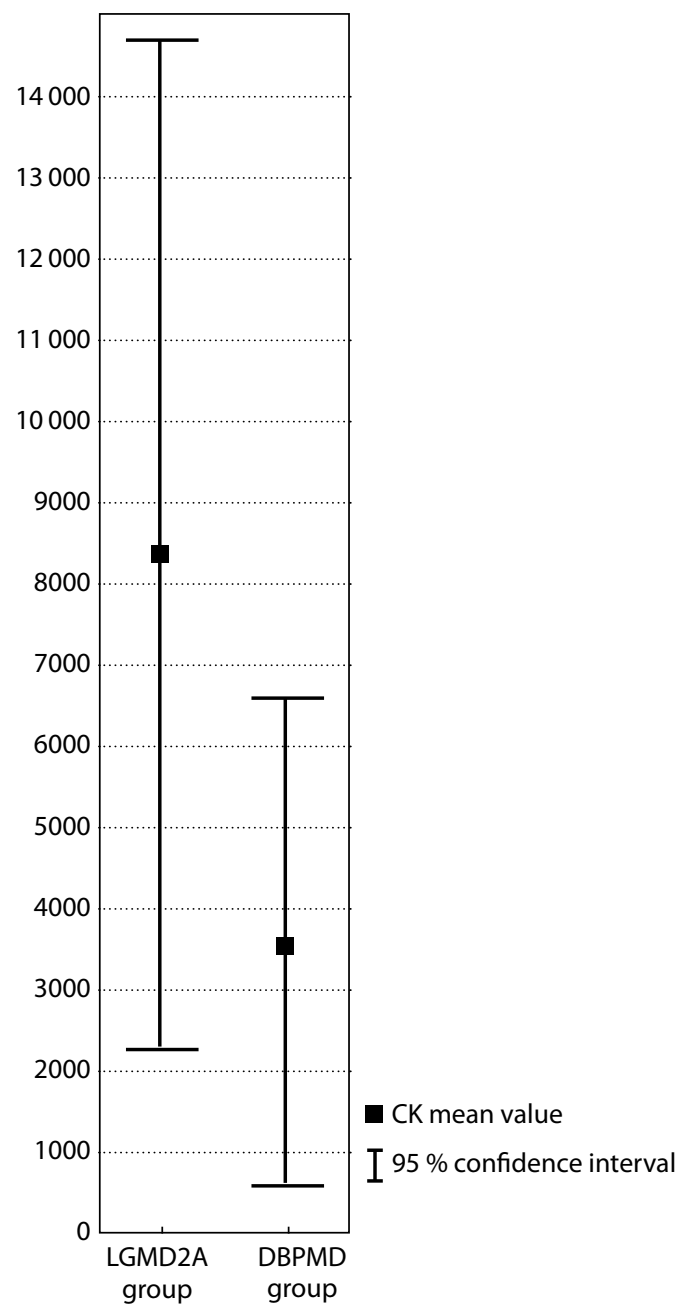

Fig. 2. Analysis of differences between the studied groups of patients according to $C K$ values tors that have a great diagnostic significance, most typical to the analyzed groups.

Thus, in DBPMD patients highly probable diagnostic signs are the following: male patients in conjunction with age of onset of the disease up to 5 years, high blood CK level at the early age and significant pseudohypertrophy of the calf muscles.

Highly probable diagnostic signs for LGMD2A patients regardless of their sex are the following: predominantly (91\% of patients) in combination age of onset 11-20 years, early formation of contractures of major joints (especially ankle joints) with walking on toes.

Thus, during the first stage of differential diagnostic of LGMD etiological factor, it is important to consider the following criteria: age of onset, sex of the patient, CK blood level, severity of pseudo hypertrophy of the calf muscles and the presence of early contractures in the ankle joints (Fig. 3).

Age of LGMD onset below 5 years

If the age of LGMD onset is below 5 years in the presence of high levels of serum CK and significant pseudohypertrophy of the calf muscles one should begin diagnostic search with looking for deletions and duplications in dystrophin gene, constituting up to $75 \%$ of all mutations in this gene. In their absence, and confidence of the doctor in the correctness of his/her diagnosis one should continue to search for point mutations in DMD gene by Sanger sequencing, or perform an exome sequencing with an analysis of all genes responsible for the occurrence of LGMD in the corresponding panel.

Autosomal recessive inheritance is most likely in case of LGMD manifestation in girls. In view of this fact and the data of our own studies, which showed that the age of onset below 5 years in this group of diseases is most prevalent in

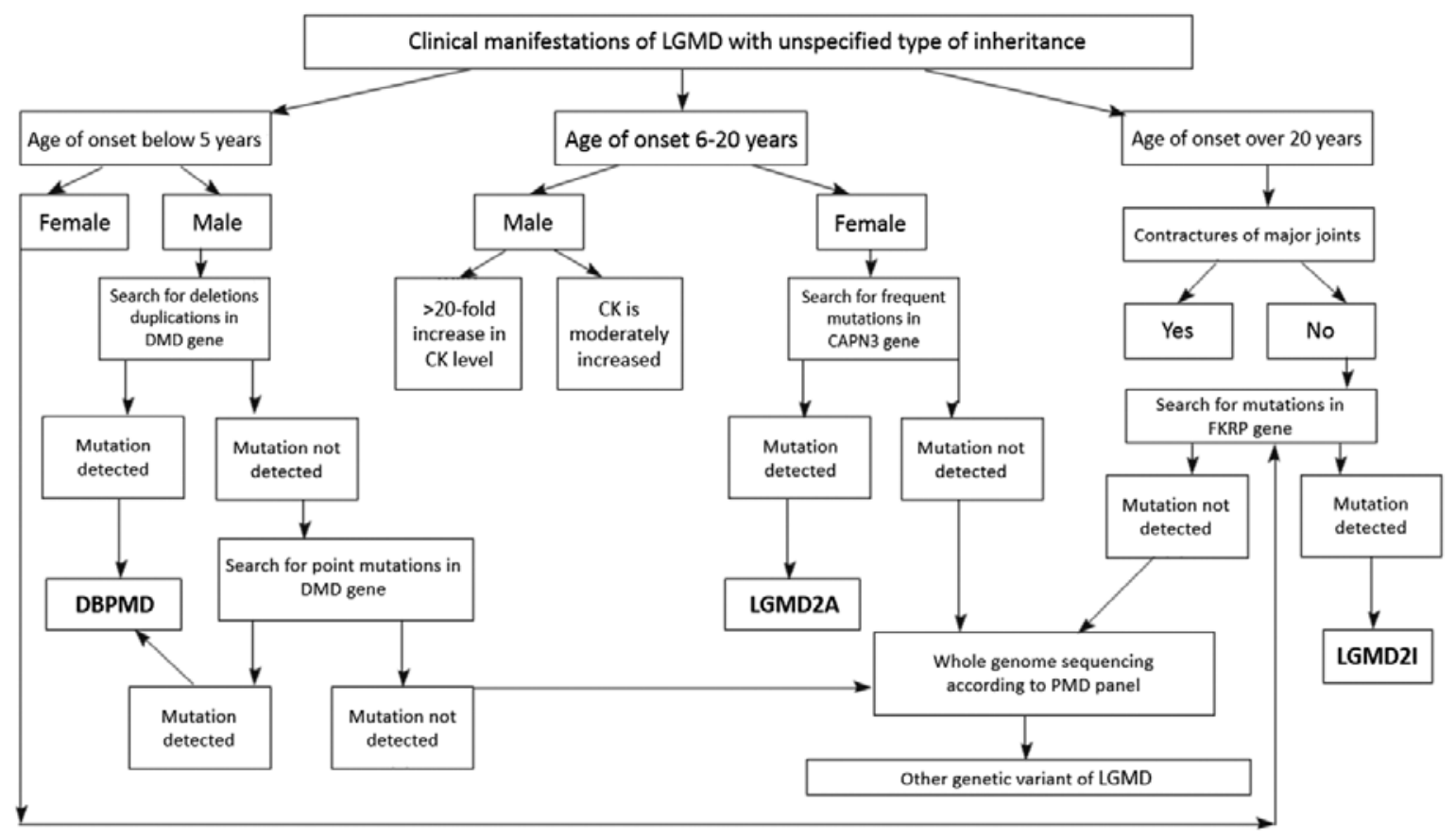

Fig. 3. Original algorithm of differential diagnosis of common genetic LGMD variants that occur after the period of normal motor development 
patients with mutations in FKRP gene responsible for the development of LGMD 2I [29], diagnostic search of etiological factor in such a case should be started from Sanger sequencing of this gene. In the absence of the desired mutations one should perform an exome sequencing analysis of all genes responsible for the developing of LGMD in the corresponding panel.

\section{Age of LGMD onset from 6 to 20 years}

In case of significant pseudohypertrophy of the calf muscles, very high CK levels in blood serum of males in this age period it is most likely to be manifestation of Becker LGMD. In this case, diagnostic search should begin with looking for deletions and duplications in the dystrophin gene. And in case of their absence and confidence of doctor in the correct diagnosis it is necessary to continue the search for point mutations in DMD gene by Sanger sequencing or exome sequencing of all genes responsible for LGMD occurrence in the corresponding panel.

However, given the data of international consortium for the study of neuromuscular diseases there are several LGMD2A phenotypes, which differ by the age of onset and degree of generalization of the process [30]. If age of onset of the disease is from 6 to 20 years and the values of CK blood levels are doubtful, regardless of sex of the patient and the presence or absence of pseudohypertrophy of the calf muscles, we recommend you to start diagnostic search with analysis of two common mutations in CAPN3 gene (550de$1 \mathrm{~A}$ and s, 598-612del), which account for $80 \%$ of all identified mutations in this gene [31-34]. In the absence of these mutations with regard to significant genetic LGMD heterogeneity it is advisable to perform whole-exome sequencing by progressive muscular dystrophy panel.

\section{Age of LGMD onset over 20 years}

The presence of contractures in the major joints is an important criterion in the first stage of diagnostic search for etiological factor in case of onset of the disease over 20 years, regardless of sex of LGMD patient. In the presence of these contractures one should start looking for common mutations in CAPN3 gene, and in case of their absence immediately begin to search for mutations in FKRP gene and only in case of negative result of the search one should continue to study the exome.

\section{R E E F}

1. Dadali E.L. Hereditary neuromuscular diseases: diagnosis and medical/genetic counseling. Author's abstract for the degree of doctor of medical sciences. M., 1999. 2. Malmberg S.A. Hereditary neuromuscular diseases in children: modern aspects of electrophysiology, diagnostics and treatment. Author's abstract for the degree of doctor of medical sciences. M, 2000.

3. Petrukhin A.S. Neurology of children age. M.: Medicine, 2004.

4. Matsumura K. LGMD2C, LGMD2D, LGMD2E, LGMD2F. Ryoikibetsu Shokogun Shirizu 2001;35:88-94.

5. Nonaka I. Muscular dystrophy: advances in research works and therapeutic trials. Rinsho Shinkeigaku 2004;44:901-4. 6. Nigro Vincenzo, Savarese Marco. Genetic basis of limb-girdle muscular dystrophies: the 2014 update. Acta Myol 2014;33(1):1-12. 7. http://neuromuscular.wustl.edu/musdist/lg. html

8. Urtasun M., Saenz A., Roudaut C. et al. Limb-girdle muscular dystrophy in Guipuzcoa (Basque Country, Spain). Brain 1998;121: $1735-47$.

9. Monckton G., Hoskin V., Warren S. Prevalence and incidence of muscular dystrophy in Alberta, Canada. Clin Genet 1982;21:19-24.

10. Emery A.E.H. Muscular Duchenne dystrophy. Oxford monographs on medical genetics. Exford: Univ. press, 1987. Vol. 15. $338 \mathrm{p}$.

11. de Paula F., Vainzof M.,

Passos-Bueno M.R. et al. Clinical variability in Calpainopathy - what makes the difference? Eur J Hum Genet 2002;10:825-32. 12. Ryzhkova O.P., Bileva D.S., Dadali E.L. et al. Clinical and genetic characteristics of limb-girdle progressive muscular dystrophy type 2A. Medical genetics 2010; 9 (11): 3-10. 13. Richard I., Broux O., Allamand V. et al. Mutations in the proteolytic enzyme calpain 3 cause limb-girdle muscular dystrophy type $2 \mathrm{~A}$. Cell 1995;27:40-4.

14. Kinbara K., Ishiura S., Tomioka S. et al. Purification of native p94, a muscle-specific calpain, and characterization of its autolysis. Biochem J 1998; 335:589-96.

15. Piluso G., Politano L., Aurino S. Extensive scanning of the calpain-3 gene broadens the spectrum of LGMD2A phenotypes. J Med Genet 2005;42(9):686-93. 16. Monaco A.P., Bertelson C.J.,

Middlesworth W. et al. Detection of deletions spanning the Duchenne muscular dystrophy locus using a tightly linked DNA segment. Nature 1985;316:842-5.

17. Monaco A.P., Neve R.L., Colletti-Feener C. et al. Isolation of candidate cDNAs for portions of the Duchenne muscular dystrophy gene. Nature 1986;323:646-50.

18. Padberg G.W. The muscular dystrophies and dystrophin. Curr Opin Neurol 1993;6(5):688-94.

19. Emery A.E. Muscular dystrophy into the new millennium. Neuromuscul Disord 2002;12(4):343-9.

20. Vainzof M., Moreira E.S., Ferraz G. et al. Further evidence for the organisation of the four sarcoglycans proteins within the dystrophin-glycoprotein complex. Eur J Hum Genet 1999;7:251-4.

21. Ueyama H., Kumamoto T., Fujimoto S. Expression of three calpain isoform genes in human skeletal muscles. J Neurol Sci 1998;155(2):163-9.

22. Anderson L.V., Harrison R.M., Pogue R. et al. Secondary reduction in calpain 3 expression in patients with limb girdle muscular dystrophy type 2B and Miyoshi myopathy (primary dysferlinopathies). Neuromuscul Disord 2000;10:553-5. 23. Canki-Klain N., Milic A., Kovac B. Prevalence of the 550delA mutation in calpainopathy (LGMD2A) in Croatia. Am J Med Genet 2004;125(2):152-6.

24. Todorova A., Georgieva B., Tournev I. et al. A large deletion and novel point mutations in the calpain 3 gene (CAPN3) in Bulgarian LGMD2A patients. Neurogenetics 2007;8(3):225-9.

25. Jones H., De Vivo D.C., Darras B.T. Neuromuscular disorders of infancy, childhood and adolescence. A clinician's approach. Oxford: Butterworth-Heinemann, 2003. 26. Brooke M.H., Fenichel G.M., Griggs R.C. et al . Clinical investigation in Duchenne dystrophy: 2, Determination of the "power" of therapeutic trials based on the natural history. Muscle Nerve 1983;6:91-103.

27. Rosalki S.B. Serum enzymes in disease of skeletal muscle. Clin Lab Med 1989;9: 767-81.

28. Zatz M., Rapaport D., Vainzof M. et al. Serum creatine-kinase (CK) and pyruvatekinase (PK) activities in Duchenne (DMD) 
as compared with Becker (BMD) muscular dystrophy. J NeurolSci 1991;102:190-6.

29. Sharkova I.V., Dadali E.L., Ryzhkova O.P., Evdokimenko V.N. Comparative analysis of the characteristics of phenotypes of limbgirdle muscular dystrophies types 2A and 2I. Neuromuscular disease in 2013; (2): 39-44.

30. Bushby K.M. Diagnostic criteria for the limbgirdle muscular dystrophies.- Report of the
ENMC Consortium on Limb-Girdle Dystrophies. Neuromuscul Disord 1995;5(1):71-4.

31. Pogoda T.V., Krakhmaleva I.N.,

Lipatova N.A. et al. High incidence of 550delA mutation of CAPN3 in LGMD2 patients from Russia. Hum Mut 2000: 15:295-300. 32. Balci B., Aurino S., Haliloglu G. et al Calpain-3 mutations in Turkey. Eur J Pediatr 2006;165:293-8.
33. Canki-Klain N., Milic A., Kovac B. Prevalence of the 550delA mutation in calpainopathy (LGMD2A) in Croatia. Am J Med Genet 2004;125(2):152-6. 34. Ryzhkova O.P. Clinical and molecular genetic analysis of the isolated limb-girdle dystrophies that are fermentopathies. Author's abstract for the degree of candidate of medical sciences. M. 2011. 


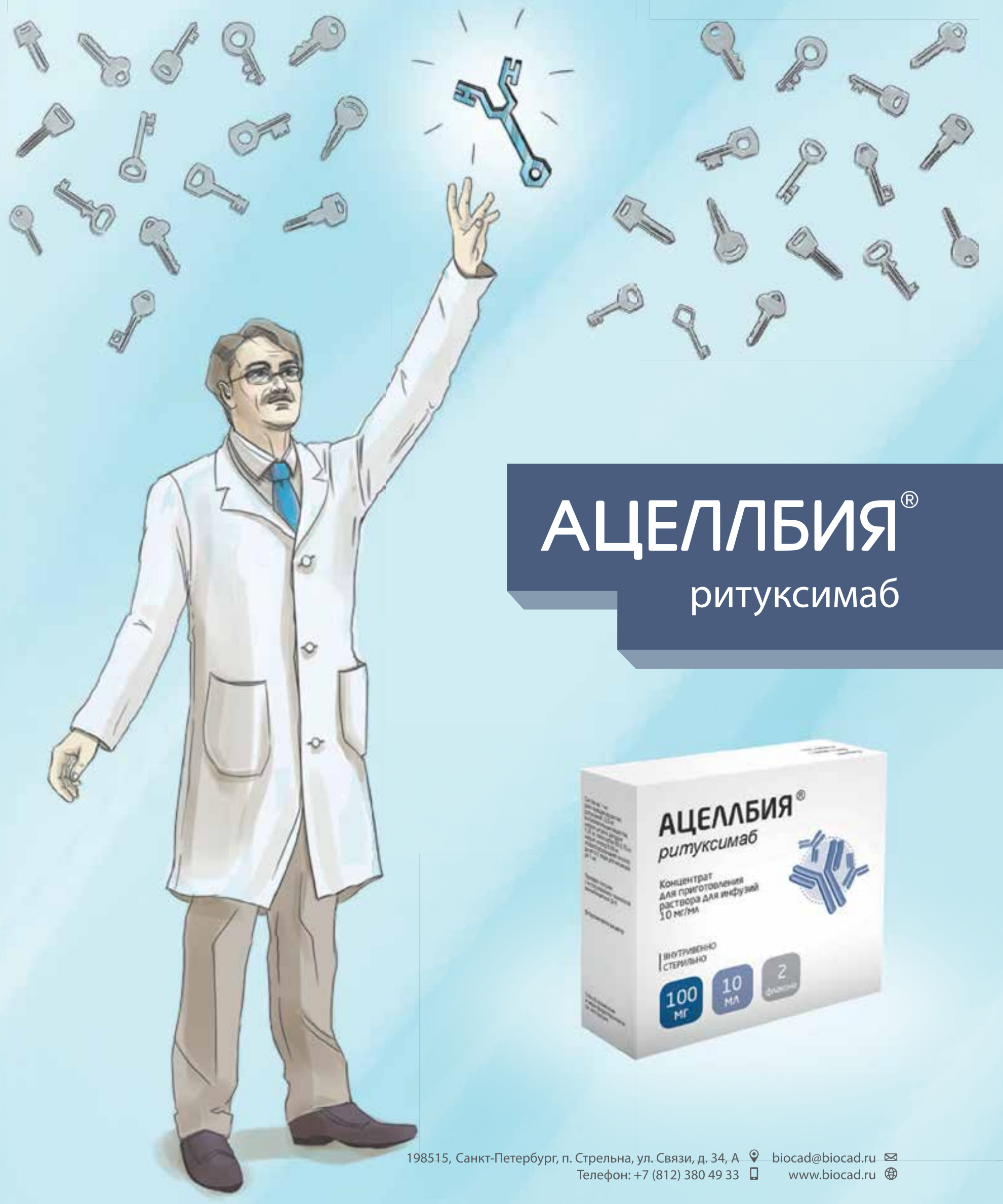

\section{BICCAD}

